\title{
Laser Processing of Dry, Wet and Immersed Polyamide Nanofiber Nonwovens with Different Laser Sources
}

\author{
Marco Götze*, Abdul Mannan Farhan**, Tobias Kürbitz**, Olaf Krimig*, \\ Sven Henning ${ }^{* * *}$, Andreas Heilmann ${ }^{* * * * *}$, Georg Hillrichs* \\ ${ }^{*}$ University of Applied Sciences Merseburg, Eberhard-Leibnitz-Str.2, 06217 Merseburg, Germany \\ georg.hillrichs@hs-merseburg.de \\ ${ }^{* *}$ University of Applied Sciences Anhalt, Bernburger Str. 55, 06266 Köthen, Germany \\ ${ }^{* * *}$ Fraunhofer Institute for Microstructure of Materials and Systems IMWS, Walter-Hülse-Str.1, 06120 Halle (Saale),
}

\begin{abstract}
Electrospun nanofiber scaffolds of different polymers are used in tissue engineering to mimic the extracellular matrices with favourable conditions for cell growth and proliferation. Structures such as cavities, holes and cuts in the scaffolds can be used to optimize cell growth. We investigated the influence of different laser sources used for direct laser writing on the cutting and structuring quality of electrospun polyamide nonwovens. Ablation thresholds and rates were determined. Because of different approaches in cell colonization with scaffolds, the investigations were carried out on dry, wet and immersed nonwovens. The results show that femto- and picosecond lasers are very well suited for processing of dry nonwovens. Processing with green wavelengths is more effective and leads to similar minimum feature sizes than in the ultraviolet range. Ablation rates up to $8000 \mu \mathrm{m}^{3} / \mathrm{pulse}$ were obtained which are about a factor of 100 higher than those for polyamide bulk material. Nanosecond UV lasers produced structures of reduced quality. Excimer lasers at $193 \mathrm{~nm}$ offer a possible alternative for large-area structures when operated at low fluences. Processing of wet and immersed nanofibers is possible with smaller processing speed and with a slightly degraded quality.

DOI: $10.2961 / \mathrm{jlmn} .2017 .03 .0019$
\end{abstract}

Keywords: picosecond laser, femtosecond laser, laser ablation, tissue engineering, polyamide nanofibers, nanofiber scaffold, electrospinning

\section{Introduction}

In regenerative medicine, damaged or malfunctioning parts of the human body are replaced by artificial tissue. By extracorporeal proliferation of autologous or xenogeneous cells on appropriate scaffolds, artificial tissue samples are generated and subsequently implanted in the human body [1-3].

To achieve effective cell growth the scaffolds used in tissue engineering should rebuild as close as possible the extracellular matrix (ECM). One promising technology for the production of cell scaffolds is electrospinning [4-7]. Fibers with diameters smaller than one micrometer are drawn from polymer solutions and form nonwovens with high surface to volume ratios and distances between the fibers, which allow the supply of the cells with nutrients. Electrospun nanofiber mats are also used as filters and membranes $[8,9]$.

To build 3D tissues the cells should migrate into the volume of the nonwovens. With spacings between the fibers typically smaller than $1 \mu \mathrm{m}$ and cell sizes of up to several $10 \mu \mathrm{m}$, growth into the fiber scaffolds is hindered. So, structuring of the nanofiber scaffolds is a way to improve the cell proliferation into the volume. For electrospun scaffolds, laser processing is one method to create holes, blind holes, slits or other geometric structures in the samples. Thermal side effects of the laser process must be avoided because melting and collapsing of the fiber network could reduce the nutrient supply of settled cells on the walls of these laser generated structures. Femtosecond (fs) lasers have been used to generate structured holes [10-13] to improve cell growth into poly (L-lactide) (PLLA), poly(vinyl alcohol) (PVA) or poly( $\varepsilon$-caprolactone) (PCL) nanofiber scaffolds. It was shown that with fs laser radiation clean structures with small heat affected zones (HAZ) could be generated and used to optimize cell population. Pulsed $\mathrm{CO}_{2}$-Lasers were also used to control structures in PCL nanofiber scaffolds and also to stack and bond thin porous nanofiber sheets together to form organ shaped 3D-scaffolds [14].

In this work, laser processing of polyamide (PA) nanofiber scaffolds as model systems with different laser sources was investigated. The aim is to find easy to handle and cost effective laser processes for cutting and structuring of nanofiber scaffolds. It was evaluated, if picosecond (ps) and nanosecond (ns) lasers with different wavelengths can be an alternative to complex fs laser systems [10-13] without reducing the quality of the laser generated features significantly. Excimer lasers are used with great success in precise micro structuring of polymers $[15,16]$. For changing of the surface properties of textile fibers excimer lasers are used $[17,18]$. Therefore, the argon fluorine excimer laser was tested as a possible source for nanofiber processing in this work

One suggested approach for the clinical use of nanofiber nonwovens is to cut small pieces or flakes with dimensions of less than $1 \mathrm{~mm}$ out of the fiber mats [19]. A suspension of these flakes can be introduced into the body by injection. It is also suggested to use these 2D flakes as a starting point to build 3D nanofiber scaffolds [19]. These flakes can be pro- 
duced in a noncontact way by laser cutting, if the heat affected zone and debris contamination is negligible and if the mechanical properties of the flakes are not degraded. To facilitate the production of flake suspensions, cutting of wet nanofiber mats is proposed. Alternatively, it was suggested to produce the flake suspension by laser cutting of nanofibers immersed in water, in saline or in a culture medium. Therefore, the feasibility of cutting and structuring of nanofiber nonwovens in dry, wet and immersed states was investigated.

\section{Material and methods}

\subsection{Electrospinning}

Through the electrospinning process, it is possible to produce polymer fibers from dissolved polymers in an electric field. Depending on the process parameters, fibers of different lengths and diameters in the nanometer range are possible. In the current work, Polyamide 6.6 (Sigma Aldrich) was dissolved in a blend of 50\% acetic acid (Carl Roth) and $50 \%$ formic acid (Carl Roth) to a concentration of $15 \%$ (w/v). The commercial electrospinning device "Nanospider" (Elmarco) was used for the electrospinning process. The process was carried out at $80 \mathrm{kV}$ with a working distance of the electrodes of $240 \mathrm{~mm}$. The temperature was kept at $22^{\circ} \mathrm{C}$ and the relative humidity was about $30 \%$.

\subsection{Laser systems}

A Nd: YAG based picosecond laser system with following specifications was used (Tab. 1).

Table 1 Data of the picosecond laser

\begin{tabular}{lc}
\hline \multicolumn{2}{c}{ Picosecond laser (Coherent: Talisker) } \\
\hline Wavelength & $355 \mathrm{~nm} \quad 532 \mathrm{~nm}$ \\
Pulse duration & $15 \mathrm{ps}$ \\
Max. pulse energy & $20 \mu \mathrm{J} \quad 40 \mu \mathrm{J}$ \\
Max. pulse repetition rate & up to $200 \mathrm{kHz}$ \\
Beam quality $\mathrm{M}^{2}$ & $<1.3$ \\
Focal spot diameter & $14 \mu \mathrm{m} \quad 23 \mu \mathrm{m}$ \\
Focusing angle (full) & $\sim 3.1^{\circ}$ \\
\hline
\end{tabular}

Alternatively, a Nd: KGW based fs laser was used in the same micromachining workstation (Tab. 2).

Table 2 Data of the femtosecond laser

\begin{tabular}{lc}
\hline \multicolumn{1}{c}{ Femtosecond laser (Light conversion: Carbide) } \\
\hline Wavelength & $343 \mathrm{~nm} \quad 515 \mathrm{~nm}$ \\
Pulse duration & $229 \mathrm{fs}$ \\
Max. pulse energy & $16 \mu \mathrm{J} \quad 26 \mu \mathrm{J}$ \\
Max. pulse repetition rate & up to $60 \mathrm{kHz}$ \\
Beam quality M ${ }^{2}$ & $<1.2$ \\
Focal spot diameter & $14 \mu \mathrm{m} \quad 23 \mu \mathrm{m}$ \\
Focusing angle (full) & $\sim 3.1^{\circ}$
\end{tabular}

A galvoscanning system (Nutfield) guided the laser beam across the sample. The sample could also be moved using a computer-controlled $x-y-z$ stage. The beam is focused by f-theta lenses ( $\mathrm{f}=100 \mathrm{~nm} @ 532 \mathrm{~nm}, \mathrm{f}=103 \mathrm{~nm}$ @ $355 \mathrm{~nm})$. Spot sizes are about $14 \mu \mathrm{m}$ at $355 \mathrm{~nm}$ and $23 \mu \mathrm{m}$ at $532 \mathrm{~nm}$.

A 355-nm nanosecond laser (Tab. 3) was used in a different micromachining workstation, in which the sample is moved on an $x-y-z-$ stage in a fixed laser beam path. Focal spot diameter is about $11.6 \mu \mathrm{m}$.

Table 3 Data of the nanosecond laser

\begin{tabular}{lc}
\hline \multicolumn{2}{c}{ Nanosecond laser (Nexlase) } \\
\hline Wavelength & $355 \mathrm{~nm}$ \\
Pulse duration & $1.9 \mathrm{~ns}$ \\
Max. pulse energy & $53 \mu \mathrm{J}$ \\
Pulse repetition rate & up to $200 \mathrm{~Hz}$ \\
Beam quality $\mathrm{M}^{2}$ & $<2$ \\
Focal spot diameter & $11.6 \mu \mathrm{m}$ \\
Focusing angle & $6.9^{\circ}$ \\
\hline
\end{tabular}

In the excimer laser experiments a mask is illuminated by the laser beam and a demagnified image of the mask is produced on the sample (Tab. 4).

Table 4 Data of the excimer laser

\begin{tabular}{lc}
\hline \multicolumn{1}{c}{ ArF Excimer laser (Coherent Compex) } \\
\hline \begin{tabular}{lc} 
Wavelength & $193 \mathrm{~nm}$ \\
Pulse duration & $20 \mathrm{~ns}$ \\
$\begin{array}{l}\text { Max. pulse energy } \\
\text { face }\end{array}$ \\
$\begin{array}{l}\text { Max. pulse repetition } \\
\text { rate }\end{array}$ & up to $100 \mathrm{~Hz}$ \\
\hline
\end{tabular}
\end{tabular}

The laser processed structures were evaluated by optical microscopy (Olympus BX50), confocal optical microscopy (OPM Messtechnik KFM) and scanning electron microscopy (LOT Phenom, FEI Quanta 3D FEG)

For comparison, also laser processing of bulk polyamide was performed (PA6.6 plate, thickness $1 \mathrm{~mm}$ and PA6.6 film, thickness $17 \mu \mathrm{m}$, from Goodfellow).

\section{Results and discussion}

\subsection{Dry nanofiber nonwovens}

\section{Ablation with solid state UV laser radiation}

To determine ablation thresholds for ns-, ps- and fs-UVsolid state laser radiation the dry polyamide 6.6 nanofiber fleece samples were irradiated at the focal plane of the focusing lens. Typical ablation spots obtained with the ps-UV laser are shown in Fig. 1. The thresholds are determined by applying the $D^{2}$-method $[20,21]$ for single pulse and in several cases also for multi pulse ablation. The diameters D of 
the laser ablated spots were measured with optical microscopy or SEM. For three solid state UV lasers, the $\mathrm{D}^{2}$ results are shown in Fig. 2 as a function of the applied fluence.

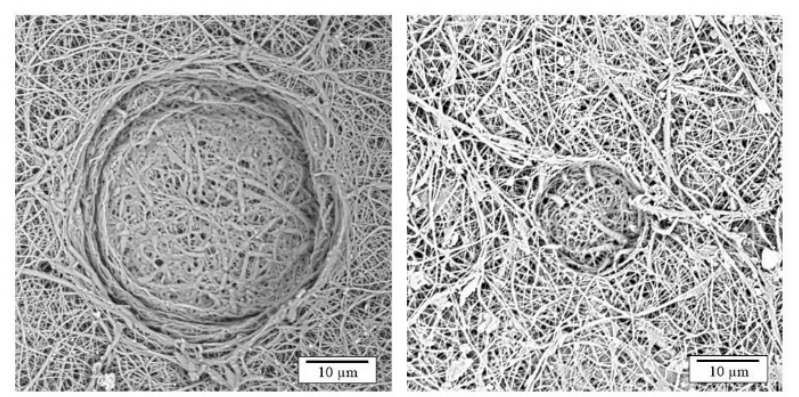

Fig. 1 Single pulse (ps-UV laser) ablation spot diameters. Left: $\mathrm{F}=11.6 \mathrm{~J} / \mathrm{cm}^{2}$, Right: $0.9 \mathrm{~J} / \mathrm{cm}^{2}$.

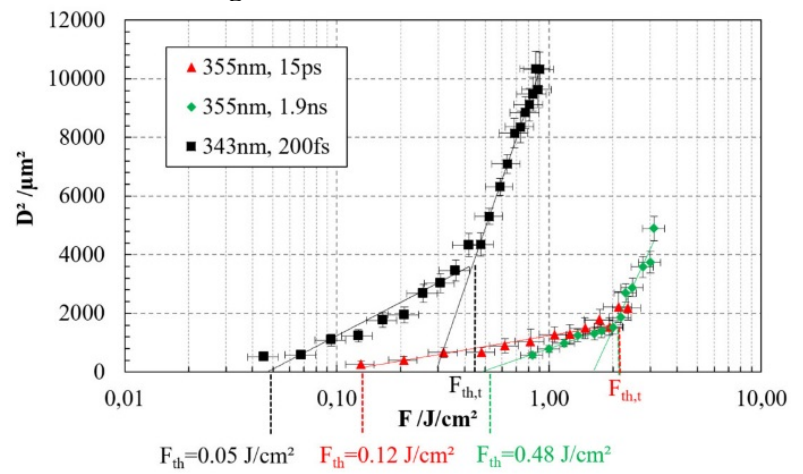

Fig. 2 Single pulse ablation threshold measurements of polyamide nanofibers with different UV laser sources. $\mathrm{D}^{2}$ of the ablated areas is plotted as a function of the laser fluence. The extrapolation of the linear fits to zero provides the ablation thresholds $\mathrm{F}_{\text {th. }}$

Table 5 Single pulse ablation thresholds (fluence and intensity) and thermal thresholds (fluence) for UV solid state lasers]

\begin{tabular}{lcc|c}
\hline \multicolumn{1}{c}{ Laser } & $\begin{array}{c}\mathrm{F}_{\mathrm{th}} / \\
\mathrm{J} / \mathrm{cm}^{2}\end{array}$ & $\begin{array}{c}\mathrm{I}_{\mathrm{th}} / 10^{10} \\
\mathrm{~W} / \mathrm{cm}^{2}\end{array}$ & $\begin{array}{c}\mathrm{F}_{\mathrm{th}, \mathrm{t} /} \\
\mathrm{J} / \mathrm{cm}^{2}\end{array}$ \\
\hline $\begin{array}{l}355 \mathrm{~nm}, \\
1.9 \mathrm{~ns}\end{array}$ & 0.48 & 0.025 & 2.1 \\
$\begin{array}{l}355 \mathrm{~nm}, \\
15 \mathrm{ps}\end{array}$ & 0.12 & 0.8 & 2.1 \\
$\begin{array}{l}343 \mathrm{~nm}, \\
229 \mathrm{fs}\end{array}$ & 0.05 & 21.8 & 0.5 \\
$\begin{array}{l}355 \mathrm{~nm}, \\
\begin{array}{l}15 \mathrm{ps}, \mathrm{PA} \\
\text { bulk }\end{array}\end{array}$ & 1.5 & 10 & $>3,5$ \\
\hline
\end{tabular}

For the ns-UV and the fs-UV laser sources two fluence ranges are found, represented by two linear fits (Fig. 2). For the ps-UV laser only few data points in the high fluence region could be measured due to the limited pulse energy of the laser source. The fluence range with the smaller slope characterizes the area of the predominantly non-thermal ablation of the material [21, 22]. Around $355 \mathrm{~nm}$ wavelength the photon energy of about $3.49 \mathrm{eV}$ is higher than the energy e.g. of C-N single bonds in polyamide of $3.16 \mathrm{eV}$ so that also photochemical ablation is possible. The threshold fluence $\mathrm{F}_{\text {th }}$ characterizes the minimum fluence to achieve material removal. For the fs-UV laser, the threshold fluence $F_{\text {th }}$ is the smallest with $0.05 \mathrm{~J} / \mathrm{cm}^{2}$. For ps-UV and ns-UV processing $F_{\text {th }}$ is 0.12 and $0.48 \mathrm{~J} / \mathrm{cm}^{2}$, respectively (Tab. 5). The focal diameter of $14 \mu \mathrm{m}$ is the same for the fs- and ps-UV-beams. Larger diameters of the ablated regions were obtained with fs-pulses than with ps-pulses (Fig. 2). The higher intensities and the lower threshold fluence enable a better utilization of the Gaussian beam wings, which leads to larger structural diameters in fs-UV ablation than for ps-UV ablation at the same fluences.

For all UV solid state laser sources, the diameters of the ablated structures are larger than the spot sizes on the sample surfaces. This behavior has also been observed in different experiments [11, 23, 24] for processing of other electrospun nonwovens with fs-lasers.

The squares of the measured diameters of the fs-UV and ns-UV processing show a second linear slope fluence range which characterizes thermal ablation (Fig. 2). For fs-UV processing, the thermal threshold fluence $F_{\text {th, }}$ is about $0.5 \mathrm{~J} / \mathrm{cm}^{2}$. For ns- and ps-single-pulse ablation, $\mathrm{F}_{\mathrm{th}, \mathrm{t}}$ is approximately $2.1 \mathrm{~J} / \mathrm{cm}^{2}$.

Previous investigations with polyamide bulk material [25] showed a distinctly higher threshold fluence of approx. $1.5 \mathrm{~J} / \mathrm{cm}^{2}$ for samples of $1 \mathrm{~mm}$ thickness. For polyamide films with a thickness of $17 \mu \mathrm{m}, \mathrm{F}_{\mathrm{th}}=4.5 \mathrm{~J} / \mathrm{cm}^{2}$ was determined. The values determined for $F_{\text {th }}$ and $F_{t h, t}$ are important for the processing of the nanofiber nonwovens to minimize thermal damage by optimizing the laser fluence used. Single-pulse ablation can already produce structures of up to $30 \mu \mathrm{m}$ depth. So, for typical thicknesses of $40 \mu \mathrm{m}$ to $80 \mu \mathrm{m}$ of the PA nonwovens laser structuring by single-pulse or few-pulse ablation is suitable.

For laser structuring of the nonwovens to optimize the cell growth or for cutting of scaffold flakes in the submillimeter range thermal damage or contamination of the samples with debris should be avoided. The HAZ was determined qualitatively from SEM images of cutting edges (Fig. $3)$.

To prevent contamination of the nonwovens with ablation products from nearby supporting structures, the samples were cut in a free-hanging way. There was no supporting structure below the sample and the flakes have to be cut with a single entity $\left(\mathrm{N}_{\mathrm{E}}=1\right)$. Otherwise, the flakes, which have not yet been completely cut, could fold away and become damaged or influence the cutting process in a non-controllable way. The fluences used in Fig. 3 were chosen, that cuts with one entity were possible. The ns-UV laser cut was made with a fluence of $21.6 \mathrm{~J} / \mathrm{cm}^{2}$ well above the thermal threshold of $2.1 \mathrm{~J} / \mathrm{cm}^{2}$. For the ps-UV-laser, a fluence of $1.42 \mathrm{~J} / \mathrm{cm}^{2}$ slightly below the thermal threshold of $2.1 \mathrm{~J} / \mathrm{cm}^{2}$ was sufficient for a cut with one entity. For the fs-UV laser the fluence of $4.14 \mathrm{~J} / \mathrm{cm}^{2}$ above the thermal threshold of $0.5 \mathrm{~J} / \mathrm{cm}^{2}$ was necessary for a cut with $N_{E}=1$. So, instead of processing with the same fluences for the different laser sources, comparable line energies $L E=f * Q / v$ (f: repetition rate, $Q$ : pulse energy, v: scanning speed) were chosen to compare the suitability of the different lasers for cutting of free hanging PA nonwovens with one entity. 


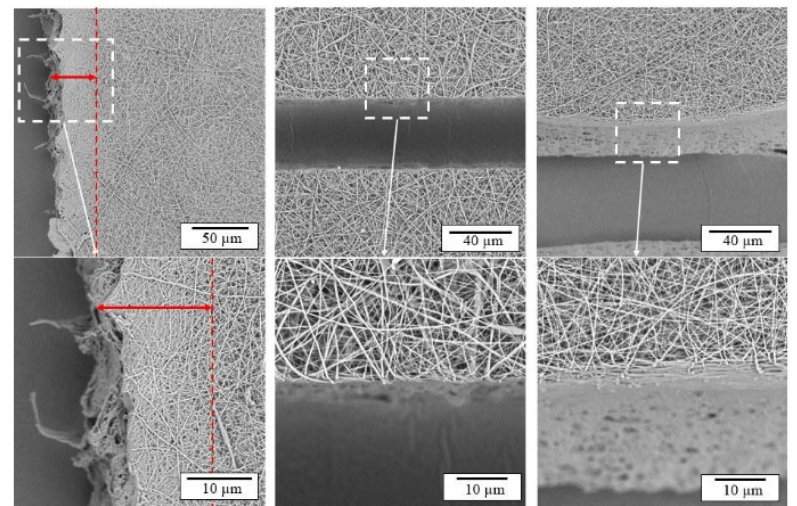

Fig. 3 Laser cuts obtained with one laser scan. The red arrows and lines indicate the zone beside the laser cut where the nanofiber structure is changed. Left: ns-UV laser (pulse energy $23 \mu \mathrm{J}$, fluence $21.6 \mathrm{~J} / \mathrm{cm}^{2} . \mathrm{v}=1 \mathrm{~mm} / \mathrm{s}$, $\mathrm{f}_{\text {rep }}=200 \mathrm{~Hz}$, line energy $\mathrm{LE}=4.62 \mu \mathrm{J} / \mu \mathrm{m})$; Center: ps-UV laser $\left(1.42 \mathrm{~J} / \mathrm{cm}^{2}, 50 \mathrm{~mm} / \mathrm{s}\right.$, $100 \mathrm{kHz}, 4.14 \mu \mathrm{J} / \mu \mathrm{m})$; Right: fs-UV laser $\left(\mathrm{F}=4.74 \mathrm{~J} / \mathrm{cm}^{2}\right.$, $100 \mathrm{~mm} / \mathrm{s}, 60 \mathrm{kHz}, 4.38 \mu \mathrm{J} / \mu \mathrm{m})$.

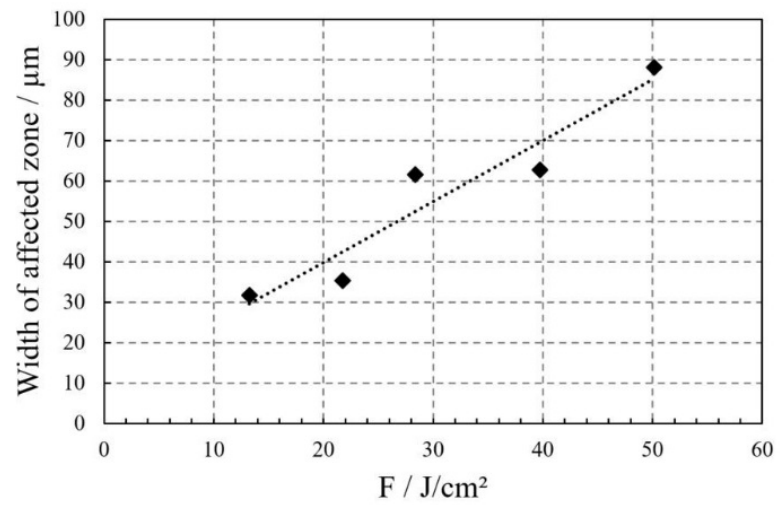

Fig. 4 Width of the nonwoven region with changed appearance in the SEM as a function of ns-UV laser fluence.

The ns-UV-processing showed laser affected zones along the cuts. A region along the cutting edge can be observed where the typical nonwoven structure (Fig. 3, red arrows) is changed. The extent of this region is fluence-dependent and increases with increasing laser fluence (Fig. 4). An explanation could be a partial melting of thinner fibers within the nonwoven, which solidify in a denser structure. Also, a contamination with very small debris particles cannot be excluded. After ps-UV- and fs-UV laser processing the cutting edges have only small zones of a few micrometer width with changed appearance of the nonwovens. No contamination with debris was detected (Fig. 3)

To determine ablation rates for the ps- and fs- lasers the depth profiles and the extensions of the laser ablated structures were measured and the ablated volumes were calculated by evaluating confocal microscopy images (Fig. 5). The nonwoven volumes ablated by single pulses are shown in Fig. 6 as a function of fluence. For comparison, the data obtained with bulk PA material are included.
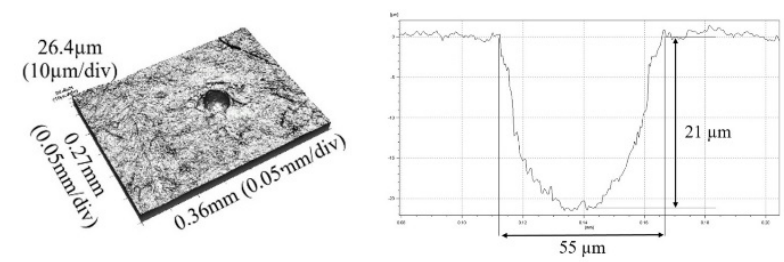

Fig. 5 Confocal image and depth profile for a typical single pulse nonwoven ablation site.

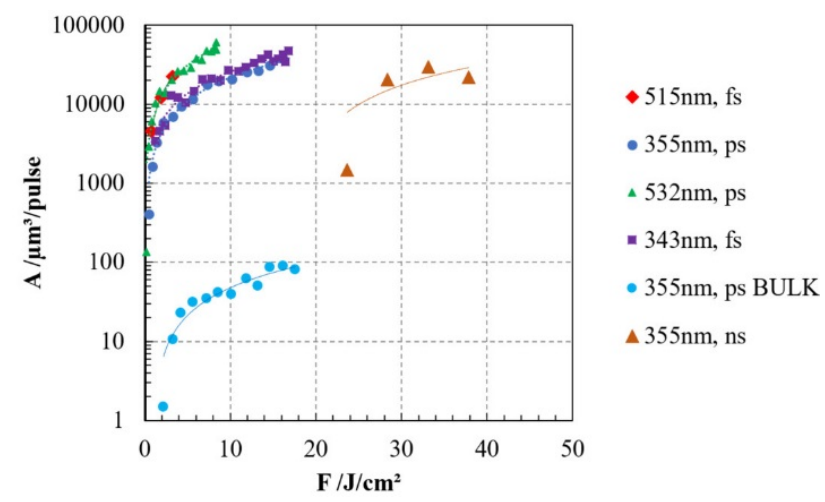

Fig. 6 Ablation rates as a function of fluence for the nonwovens and the PA bulk material.

For all solid state laser sources the ablated nonwoven volume increases with the fluence. The volumes ablated by the ps-UV and fs-UV-lasers lasers are nearly identical within the experimental errors. A greater average depth was obtained with ps-UV radiation than with fs-UV radiation. However, due to the significantly larger ablation diameters for the fs-UV laser, the ablation rates turn out to be similar. For bulk material, the ablation rates are about two orders of magnitude smaller.

To estimate the density $\rho_{\mathrm{nw}}$ of the nonwovens, samples with $2 \mathrm{~cm} \times 2 \mathrm{~cm}$ areas were balanced. The thickness of the samples (about $80 \mu \mathrm{m}$ ) was measured by confocal microscopy. It was found that $\rho_{\mathrm{nw}}$ is about $0.09 \mathrm{~g} / \mathrm{cm}^{3}$. For bulk PA 6.6 a density $\rho_{\text {B }}$ of $1.13 \mathrm{~g} / \mathrm{cm}^{3}$ was determined. The porosity $\mathrm{p}_{\mathrm{nw}}$ [26] of the nonwoven PA 6.6 was calculated by $\mathrm{p}_{\mathrm{nw}}=\left(\rho_{\mathrm{B}}-\rho_{\mathrm{nw}}\right) / \rho_{\mathrm{B}}$ and found to be about $92 \%$.

The high ablation rates for the nanofiber samples are probably due to the high porosity, the lower ablation thresholds (Tab. 5), and the light diffusion into the porous nonwovens. Together, these effects result in ablation in a more extended area than in the bulk material. To obtain similar ablation rates as for the ultrashort UV lasers the ns-UVlaser must be operated at higher fluences.

\section{Excimer laser processing of dry PA nonwovens}

In addition to the processing with solid-state laser pulses of different pulse durations, experiments were also carried out with an ArF excimer laser. Typical PA nanofiber fleece ablation sites obtained by the excimer laser are shown in Fig. 7 for three different fluences. For comparison, in Fig. 7 also the ablation of PA bulk material is shown. 

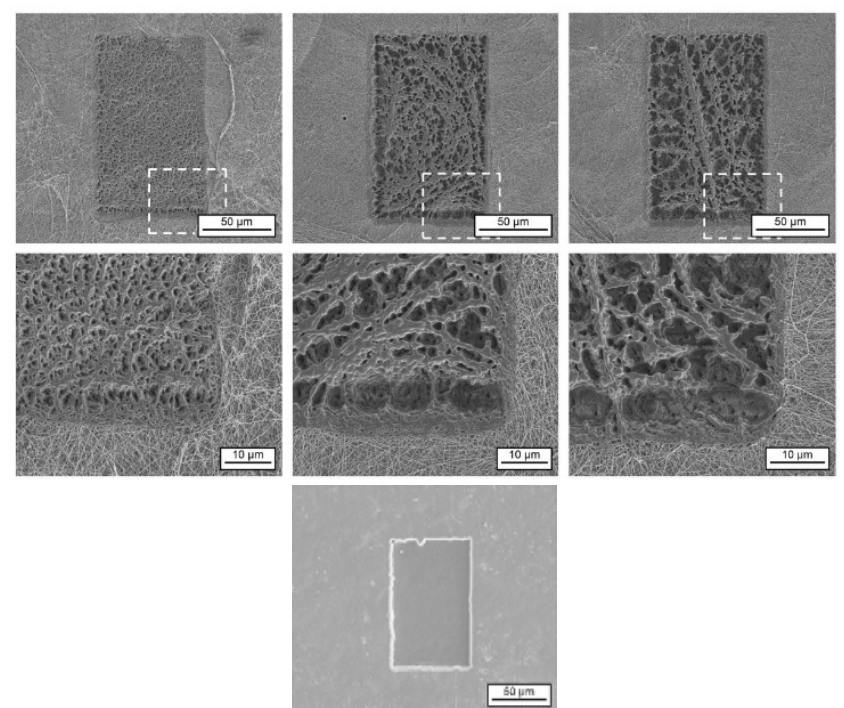

Fig. 7 Nanofiber fleece ablation spots after 50 excimer laser pulses. Top: $\mathrm{F}=14 \mathrm{~mJ} / \mathrm{cm}^{2}, 33 \mathrm{~mJ} / \mathrm{cm}^{2}$ and $43 \mathrm{~mJ} / \mathrm{cm}^{2}$. Below: Enlarged sections as indicated by the white squares. Bottom: PA bulk ablation, $\mathrm{F}=430 \mathrm{~mJ} / \mathrm{cm}^{2}$.

To estimate the ablation thresholds for excimer laser processing of the fleece material structures were processed with different fluences and analysed by optical microscopy. The fluence between the smallest, with visible ablation, and the highest, without visible ablation, is taken as the ablation threshold. In Fig. 8 the data are collected and compared with the results obtained with bulk PA 6.6.

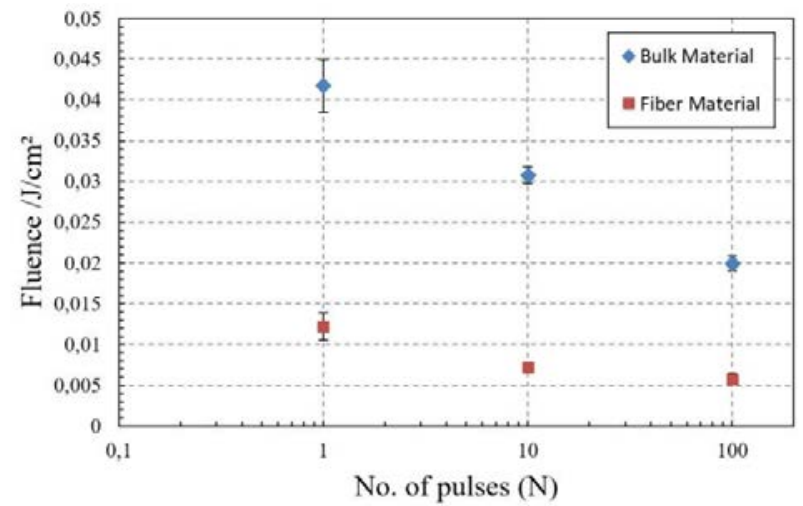

Fig. 8 Estimated thresholds for excimer laser ablation of PA nanofiber fleece and PA bulk material for single and multi-pulse ablation.

Single pulse and multi pulse ablation thresholds for the PA bulk material are higher than the thresholds for the nanofiber material. Probably, the polymer bonds in the nanofibers are partly weakened by the electrospinning process. Also, light scattering in the fiber mats may result in a more effective interaction between the laser photons and the polymer than in the bulk material. The decrease of the ablation threshold with the number of laser pulses is due to creation of absorbing sites by the first laser pulses. A similar incubation behaviour was observed by Krüger et al. [27] for $248 \mathrm{~nm}$ ablation of polycarbonate, while for polyimide and polyethylene terephthalate no incubation was observed. Sharp edges of the ablated spots on the nanofiber fleece are obtained (Fig. 7). On the bottom of the areas irradiated by
50 pulses at a fluence of $14 \mathrm{~mJ} / \mathrm{cm}^{2}$, a change in the fiber structure was observed. The nanofibers tend to collapse and to form thicker fibers. This tendency increases with increasing fluence. In contrast, PA bulk ablation (Fig. 7, bottom) is possible with sharp edges and with no observable thermal damage.

Yip et al. [28] studied $193 \mathrm{~nm}$ ablation on PA 6 textile fibers of about $15 \mu \mathrm{m}$ diameter. For fluences above 13 $\mathrm{mJ} / \mathrm{cm}^{2}$ they observed ripple formation on the fibers. Comparable to the nanofiber merging observed in this work the tiny ripples on the textile fibers merge together with increasing number of pulses. The penetration depth of $193 \mathrm{~nm}$ radiation in nylon 6 is about $0.5 \mu \mathrm{m}$ [29] and larger than the diameters of the nanofibers. Taken into account the results of Ref. [29] it is expected that the temperature throughout the nanofibers increases above the melting temperature already at moderate fluences, while for the thicker textile fibers only a surface layer is molten.

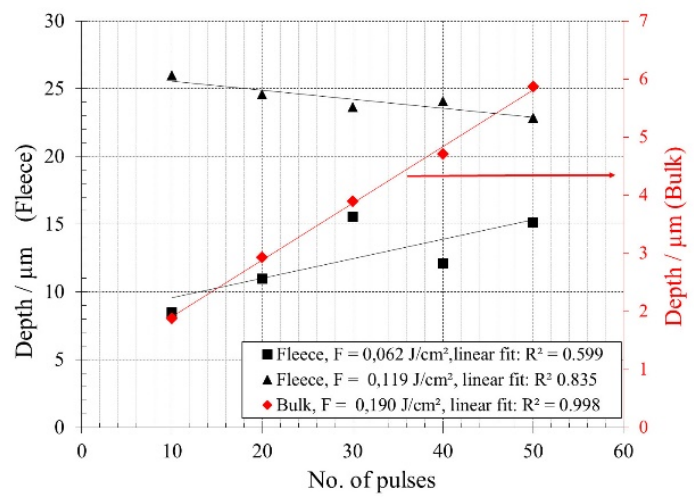

Fig. 9 Ablation depth for excimer laser ablation of PA nanofiber fleece for two different fluences and for PA bulk material. The repetition rate was $100 \mathrm{~Hz}$.

For the PA nanofiber fleece, it was found that the ablation depth is increasing with the fluence, but that it is not increasing within the experimental errors with increasing pulse numbers (Fig. 9). For PA bulk material, the ablation depth increases linear with the number of pulses. It is assumed that the surface structure of the fleece is altered already by the first laser pulses. The applied intensity of the following pulses is not sufficient to ablate the changed surface. So, nonwoven ablation control by the number of excimer pulses is hard to achieve.

\section{Ablation with green fs and ps lasers}

In addition to nanofiber processing with laser sources in the UV range, investigations were carried out in the green wavelength range at $515 \mathrm{~nm}$ and $532 \mathrm{~nm}$ with pulse durations of 229 fs and 15 ps, respectively. The ablation thresholds are derived from the data shown in Fig. 10 and are collected in Tab.6. For single pulse ablation of dry nonwovens, similar ablation thresholds were determined as for UV laser processing (Tab. 5): $\mathrm{F}_{\text {th }}=0.14 \mathrm{~J} / \mathrm{cm}^{2}(532 \mathrm{~nm}, 15 \mathrm{ps}), \mathrm{F}_{\text {th }}=$ $0.07 \mathrm{~J} / \mathrm{cm}^{2}$ ( $\left.515 \mathrm{~nm}, 229 \mathrm{fs}\right)$. Thus, the effect of direct bond breaking by UV photons seems to have little effect on the ablation threshold. For the green wavelengths, the thermal thresholds $\mathrm{F}_{\mathrm{th}, \mathrm{t}}$ of $2.9 \mathrm{~J} / \mathrm{cm}^{2}$ (532nm, 15ps) and $1.1 \mathrm{~J} / \mathrm{cm}^{2}$ (515 nm, $229 \mathrm{fs}$ ) are estimated from the data shown in Fig. 
10. The "green" thermal ablation thresholds are slightly higher than those in the UV range.

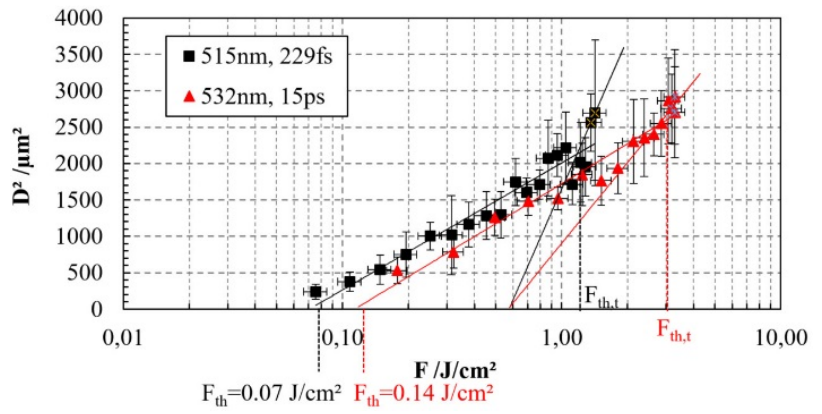

Fig. 10 Single pulse ablation threshold measurements of polyamide nanofibers with $515 \mathrm{~nm}$ fs- and 532nm ps-laser.

Table 6 Single pulse ablation thresholds (fluence and intensity) and thermal thresholds (fluence) for green solid state lasers.

\begin{tabular}{ccc|c}
\hline Laser & $\begin{array}{c}\mathrm{F}_{\mathrm{th}} / \\
\mathrm{J} / \mathrm{cm}^{2}\end{array}$ & $\begin{array}{c}\mathrm{I}_{\mathrm{th}} / \\
10^{10} \mathrm{~W} / \mathrm{cm}^{2}\end{array}$ & $\begin{array}{c}\mathrm{F}_{\mathrm{th}, \mathrm{t}} / \\
\mathrm{J} / \mathrm{cm}^{2}\end{array}$ \\
\hline $532 \mathrm{~nm}, 15 \mathrm{ps}$ & 0.14 & 0.93 & 2.9 \\
$515 \mathrm{~nm}, 229 \mathrm{fs}$ & 0.07 & 31 & 1.1 \\
\hline
\end{tabular}

The diameters of the structures produced with green fspulses are slightly larger than those produced with green pspulses. The wings of the fs beam profile contribute to the ablation in an extended area because of the higher intensity for these pulses (Fig. 10).

Although the focal spot sizes of the green and the UV laser are different (UV: $14 \mu \mathrm{m}$, green: $23 \mu \mathrm{m}$ ) it was found that the sizes of the ablated structures with the green ps laser are comparable to the sizes obtained with the ps-UV laser. So, it turns out that it is not possible to achieve finer structures on the nanofiber nonwovens with UV laser light than with green laser radiation. It is assumed, that the interplay of wavelength dependent light scattering in the strongly inhomogeneous mats with fiber diameters and spaces in the range of the wavelengths and different ablation thresholds result in larger laser affected areas for UV laser sources.

During cutting or structuring of PA nonwovens with the green lasers, small HAZs of less than $5 \mu \mathrm{m}$ (532 nm, $15 \mathrm{ps)}$ and less than $2 \mu \mathrm{m}$ (515 nm, $229 \mathrm{fs)}$ are formed (Fig. 11). The cuts shown were obtained with a single scan line $\left(\mathrm{N}_{\mathrm{E}}=1\right)$. The applied line energy LE was similar for both lasers.

The ablation rates for the green ps-laser and the green fslaser are nearly identical and higher than those for the UV solid state lasers. This is consistent with the calculated optical penetration depth $\alpha_{e f f}^{-1}$ obtained by evaluation of the measured hole depths h per pulse. A phenomenological Lambert-Beer model [30,31] was used to calculate $\alpha_{\text {eff }}^{-1}$ :

$$
h=\alpha_{\text {eff }}^{-1} \cdot \ln \left(\frac{F}{F_{t h}}\right)
$$

$F$ is the fluence and $F_{\text {th }}$ the threshold fluence. Where possible, the data have been analyzed for the low fluence range $\left(2 * \mathrm{~F}_{\text {th }}<\mathrm{F}<0.5 \mathrm{~F}_{\mathrm{th}, \mathrm{t}}\right)$ and for the high fluence range $(\mathrm{F}>$ $\left.2 * F_{t h, t}\right)$. In the high fluence range, the penetration depth for the green fs and the green ps laser is about $27 \mu \mathrm{m}$. In the low fluence range, the penetration depth is about $12 \mu \mathrm{m}$.
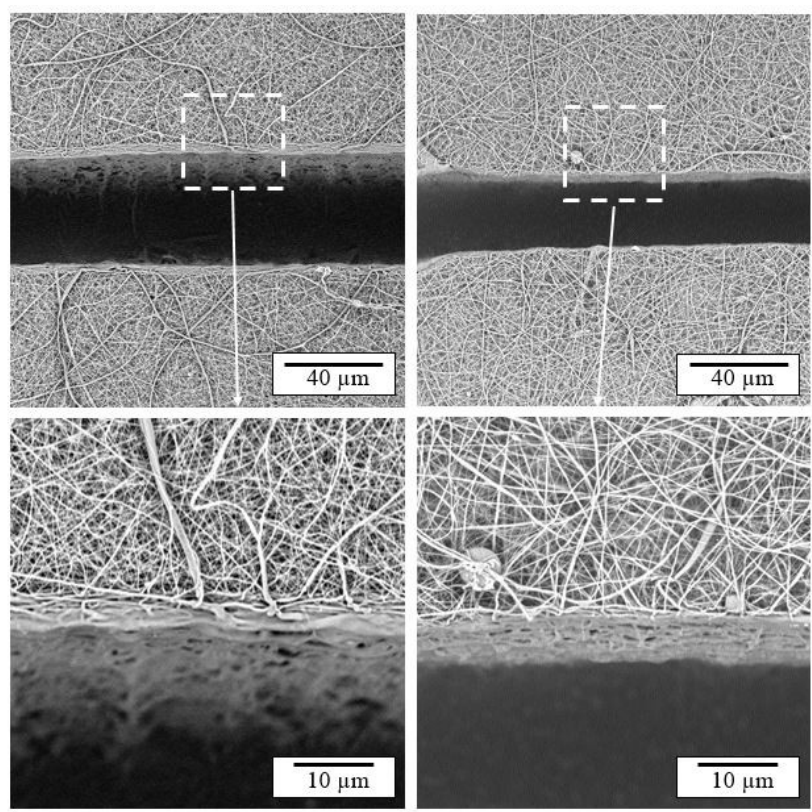

Fig. 11 SEM images of dry nanofiber flake cuts obtained with one scan line. The white squares indicate the magnified areas shown below. Left: ps-laser ablation, $\lambda=532 \mathrm{~nm}, \mathrm{~F}=0.81 \mathrm{~J} / \mathrm{cm}^{2}$, $\mathrm{v}=200 \mathrm{~mm} / \mathrm{s}, \mathrm{f}_{\mathrm{Rep}}=200 \mathrm{kHz}, \mathrm{LE}=4 \mu \mathrm{J} / \mu \mathrm{m}$. Right: fs-laser ablation, $515 \mathrm{~nm}, 0.79 \mathrm{~J} / \mathrm{cm}^{2}, 50 \mathrm{~mm} / \mathrm{s}, 60 \mathrm{kHz}, 3.6 \mu \mathrm{J} / \mu \mathrm{m}$.

For the ps-UV laser, in the high fluence range, the penetration depth is about $14 \mu \mathrm{m}$, while for the fs-UV-laser the corresponding penetration depth is only $5.5 \mu \mathrm{m}$. As mentioned above, because of the bigger ablation spot diameters for the fs-UV laser, the ablated volumes are similar for these lasers (Fig. 6). For PA 6.6 bulk material the multi pulse thermal threshold was estimated to be higher than about 3.5 $\mathrm{J} / \mathrm{cm}^{2}$. So, a penetration of less than $0.9 \mu \mathrm{m}$ in the high fluence range and less than $0.2 \mu \mathrm{m}$ in the low fluence range was estimated. Because of smaller ablation spot diameters and smaller penetration depths, the ablated volumes of the bulk material are much smaller than those for the nanofiber nonwovens.

The $355 \mathrm{~nm}$ photon energy $\mathrm{E}_{\mathrm{P}}$ of $3.49 \mathrm{eV}$ is higher than the C-N-bond energy of $3.16 \mathrm{eV}$ in the PA 6.6 polymer, so that ablation by direct bond breaking is possible. For the 515 $\mathrm{nm}\left(\mathrm{E}_{\mathrm{p}}=2.41 \mathrm{eV}\right)$ and $532 \mathrm{~nm}\left(\mathrm{E}_{\mathrm{p}}=2.33 \mathrm{eV}\right)$ the photon energy is not sufficient for $\mathrm{C}-\mathrm{N}$ bond breaking, so that nonlinear absorption is necessary for ablation.

So, comparing Fig. 3 and Fig. 11 it is obvious that, with moderate fluences, high cutting edge qualities can be achieved with the UV and also with the green ultrashort pulse lasers. The UV ns-laser cuts are of a somewhat reduced quality. The green ps-laser is an efficient tool for precise cutting of dry PA nonwoven and favourable because of the less complexity compared to the fs- and the UV lasers.

Using mask imaging the 193-nm excimer laser is useful for larger area processing of dry nonwovens, however the fluence must be carefully controlled to avoid thermal effects in the irradiated areas (Fig. 7). 


\subsection{Wet nanofiber nonwovens}

In certain application scenarios, it is necessary to produce nanofiber scaffolds from wet samples, e.g. cutting of populated nonwovens when they are soaked with a nutrient medium, with a PBS solution or with deionized water. To cut wet nanofiber nonwovens the samples were placed in deionized water for $30 \mathrm{~min}$. Then, the samples were taken out of the water, drops were removed, and then the wet nonwovens were immediately processed by the laser. The Ablation thresholds are obtained from $\mathrm{D}^{2}(\mathrm{~F})$ measurements as shown in Fig. 12. The results are collected in Table 7.

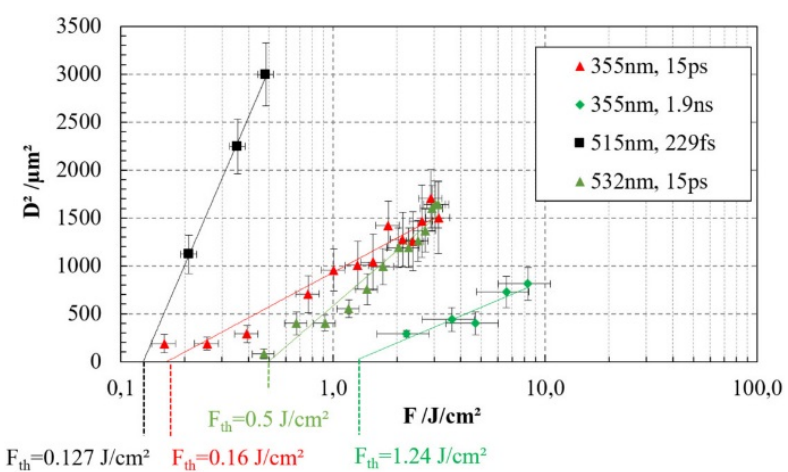

Fig. 12 Determination of single pulse ablation thresholds for wet nanofiber fleece samples

Table 7 Single pulse ablation threshold for wet PA nanofiber fleece. *: Data taken from [25] , **: this work

\begin{tabular}{ccc}
\hline \multicolumn{1}{c}{ Laser } & $\begin{array}{c}\mathrm{F}_{\mathrm{th}} / \\
\mathrm{J} / \mathrm{cm}^{2}\end{array}$ & $\begin{array}{c}\mathrm{I}_{\mathrm{th}} / \\
10^{10} \mathrm{~W} / \mathrm{cm}^{2}\end{array}$ \\
\hline $355 \mathrm{~nm}, 1.9 \mathrm{~ns}^{* *}$ & 1.24 & 0.12 \\
$355 \mathrm{~nm}, 15 \mathrm{ps}^{*}$ & 0.16 & 1.06 \\
$515 \mathrm{~nm}, 229 \mathrm{fs} * *$ & 0.127 & 30.5 \\
$532 \mathrm{~nm}, 15 \mathrm{ps}^{* *}$ & 0.5 & 0.93 \\
\hline
\end{tabular}

The ablation thresholds for the wet nonwovens are higher than those obtained for ablating dry samples (Tab. 5 and Tab. 6).
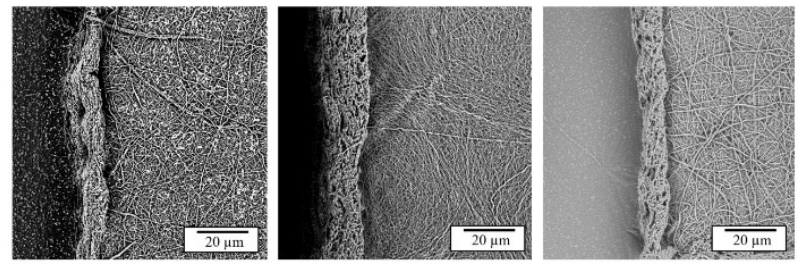

Fig. 13 SEM Images of processed wet nanofiber flakes. Left: nsUV-ablation $\mathrm{F}=37.1 \mathrm{~J} / \mathrm{cm}^{2}, \mathrm{v}=1 \mathrm{~mm} / \mathrm{s}, \mathrm{f}_{\mathrm{rep}}=200 \mathrm{~Hz}$, number of laser scans $\mathrm{N}_{\mathrm{E}}=1$, line energy $\mathrm{LE}=8.4 \mu \mathrm{J} / \mu \mathrm{m}$; Center: ps-UV-ablation $\left(5.4 \mathrm{~J} / \mathrm{cm}^{2}, 200 \mathrm{~mm} / \mathrm{s}, 200 \mathrm{kHz}, \mathrm{N}_{\mathrm{E}}=1,8.0 \mu \mathrm{J} / \mu \mathrm{m}\right)$; Right: fsUV-ablation $\left(8.61 \mathrm{~J} / \mathrm{cm}^{2}, 100 \mathrm{~mm} / \mathrm{s}, 60 \mathrm{kHz}, \mathrm{N}_{\mathrm{E}}=1,7.5 \mu \mathrm{J} / \mu \mathrm{m}\right)$.

In Fig. 13, cutting results of wet samples with the different UV solid state lasers operated at similar line energies LE are shown. Compared to dry UV laser processing, larger laser affected zones are formed at the cutting edges after "wet" processing with ns-UV, ps-UV and fs-UV irradiation. It also appears that the top layers of the nonwovens tend to delam- inate and to curl after wet cutting. This has already been observed in ps-UV processing [32]. The fine fiber structure of the nonwovens in the curls is changed. Comparing the results shown in Fig. 3 for processing of dry nonwovens with those shown in Fig. 13 for wet samples, it is obvious that the presence of water in the wet samples strongly changes the cutting process. The wet samples were processed with intensities of about $2 * 10^{10} \mathrm{~W} / \mathrm{cm}^{2}(\mathrm{~ns}), 2 * 10^{11} \mathrm{~W} / \mathrm{cm}^{2}(\mathrm{ps})$ and $4 * 10^{12} \mathrm{~W} / \mathrm{cm}^{2}$ (fs). These intensities are close to or above the intensity at which the transmission of pure deionized water drops by laser-induced breakdown (cf. Fig. 16), plasma absorption, beam scattering and deflection by cavitation bubbles. Also the threshold for laser induced breakdown (LIB) may decrease by increased laser absorption due contamination of the water by fiber ablation debris. As mentioned above, the dry PA nanofiber nonwovens have a porosity of about $92 \%$ and take up water of about 13 times of the their own weight. The water intake of the PA 6.6 polymer itself is small (2.3 \% within $24 \mathrm{~h}$, according to the specifications of the supplier of bulk PA), so the water mainly fills the spaces between the nanofibers. Probably, the bigger fraction of the laser energy is absorbed in the water filled spaces and the fibers are partly shielded from direct laser irradiation. It is known that the destruction or lysis of biological cells under a water layer [33] by ns laser radiation is mainly due to cavitation bubbles created by LIB in the water above the cell layer. Thus, it is assumed that similar processes driven by LIB occur in laser cutting of wet nanofiber nonwovens. Cavitation bubble dynamics are also typically accompanied by a temperature increase [34]. Also the remaining laser energy absorbed at fluences below the ablation threshold will increase the temperature and can contribute to partly melting and collapsing of fibers along the cut edges. So, higher thresholds for processing of wet nonwovens are attributed to water absorption.

It has to be considered, that during preparation for the scanning electron microscope the fiber samples cut in the wet state have to be dried. Drying of the nonwovens is accompanied by a certain shrinking of the samples, but we did not observe the formation of curls after drying of wet samples cut mechanically by a scalpel. So the curls observed in Fig. 13 are probably formed during the laser process.

There are only small differences in the morphology of the cut edges for the wet samples (Fig. 13) obtained with the ns-, ps,- and fs-laser and nearly the same line energy. This is an indication that cutting of wet nonwovens is driven by LIB in the water surrounding the nanofibers. On the contrary, the cuts of the dry nonwovens (Fig. 3) are generated by direct, pulse duration dependent, laser ablation of the polyamide.

For structures generated with the ps-UV laser, e.g. holes or channels, even at moderate fluences partly fusion of the fibers in the upper layers were observed on the bottom of the structures. The different surface texture could possibly counteract a successful cell colonization. Using the fs-UV laser fiber fusion is strongly reduced. Even smaller thermal laser effects on the fiber morphology have been observed for processing with the green fs-laser. As seen in Fig. 14 there is an intact structure with a slightly reduced packaging of the fibers on the bottom of the fabricated structure, but with negligible thermal damage. Like the results in the UV range (Fig 13), a small laser affected zone is formed on the cutting edges. 

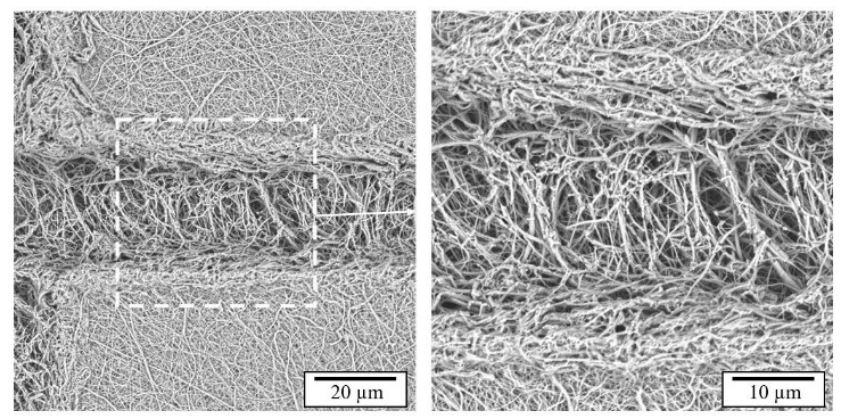

Fig. 14 Laser generated grooves in wet fleece samples. Left: green fs-radiation $\left(\mathrm{F}=1.93 \mathrm{~J} / \mathrm{cm}^{2}, \mathrm{f}_{\text {rep }}=60 \mathrm{kHz}, \mathrm{v}_{\mathrm{scan}}=400 \mathrm{~mm} / \mathrm{s}\right)$; Right: magnified image of the marked area.

The ablation rates for the green laser sources with up to $8000 \mu \mathrm{m}^{3}$ / pulse (Fig. 15) are well below the rates for dry processing (Fig. 6). There are only small differences between ps- and fs-processing. For ns-UV processing of wet samples higher fluences must be applied to achieve similar ablation rates. This tendency was also observed for dry samples (Fig. 6).

The smallest diameter of ablation spots of about $10 \mu \mathrm{m}$ for "wet "cutting was obtained with the green ps-laser. For comparison, the green fs-laser produced minimum spot sizes of about $33 \mu \mathrm{m}$. In general, the ablation depths for wet processing are smaller than those for dry processing.

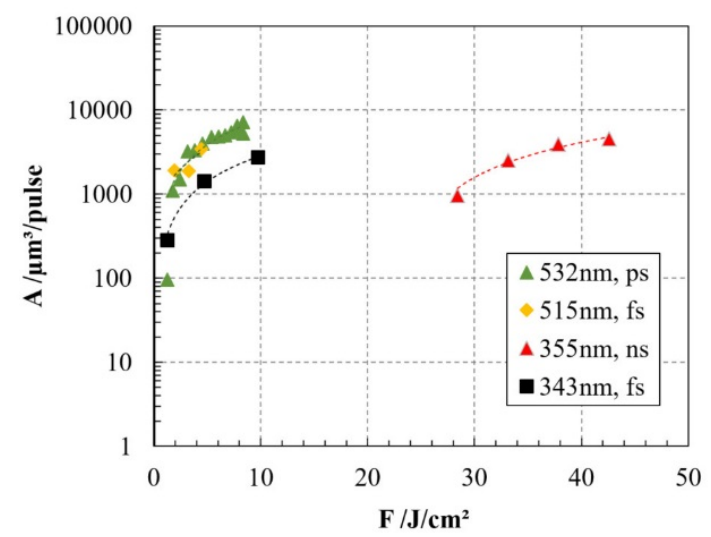

Fig. 15 Ablated volumes of wet samples as a function of fluence.

\subsection{Immersed nanofiber nonwovens}

For special applications [19] it is necessary to produce nanofiber flakes from nonwovens immersed in liquids as nutrient media, PBS solutions or deionized water.

To evaluate useful fluence and intensity ranges for structuring and cutting of immersed PA nanofiber samples the transmission of deionized water (Fig. 16) was measured for the UV laser wavelengths $355 \mathrm{~nm}$ (ns- and ps-laser) and $343 \mathrm{~nm}$ (fs-laser). The laser beams were guided through a 10 -mm water column in a fused silica petri dish. As indicated in Fig. 16 in the low intensity range several data were taken with the water column out of focus. In these cases, the laser spot size on the water surface was used for intensity calculations. For higher intensities of the ns- and ps-laser the focus was placed about $3 \mathrm{~mm}$ below the water surface and the focal spot size was taken for intensity calculations.

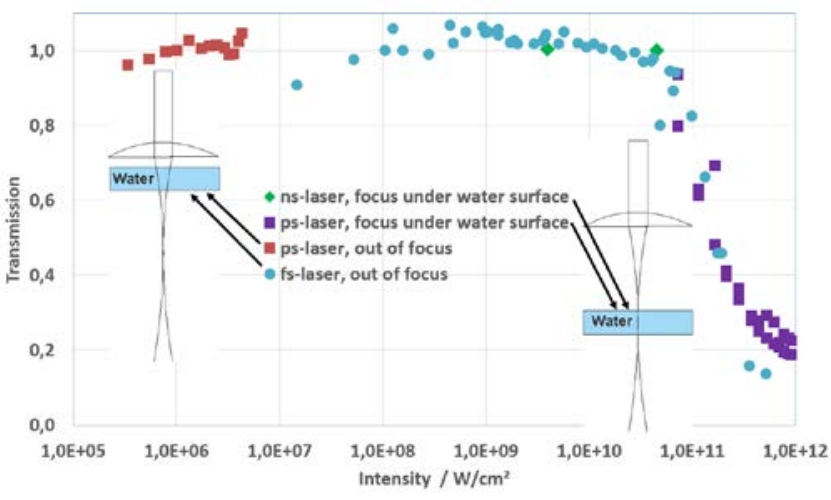

Fig. 16 Transmission of a column of deionized water of $10 \mathrm{~mm}$ height for different UV solid state laser. The beam paths are indicated in the figure.

For moderate intensities below $1 * 10^{11} \mathrm{~W} / \mathrm{cm}^{2}$ the linear absorption is negligible. Above this limit, the transmission of the ps- and fs- UV-laser beams become unstable and drops rapidly. This is attributed to laser-induced breakdown in water followed by plasma absorption and disturbing the beam by cavitation bubble formation. The threshold intensity for the optical breakdown estimated here for the ps- and fs- UV laser beams is in the same order of magnitude as those found by Vogel et al. [35] for $532 \mathrm{~nm}-60 \mathrm{ps}$ pulses $\left(2.8 * 10^{11} \mathrm{~W} / \mathrm{cm}^{2}\right)$, and lower than those observed for 580 $\mathrm{nm}-300 \mathrm{fs}$ pulses $\left(48^{*} 10^{11} \mathrm{~W} / \mathrm{cm}^{2}\right)$. For ps- and fs-UV lasers no threshold data were available for direct comparison, it is assumed that the ultrashort pulses at the UV wavelengths used in this work will lead to a more efficient multiphoton absorption and therefore to a lower optical breakdown threshold compared to the thresholds for green wavelengths.

For the ns-, ps- and fs-UV solid state lasers used in this work the maximum power is $0.07 \mathrm{MW}, 1.7 \mathrm{MW}$ and 206 MW respectively. The critical power for self-focusing is given by [36] with the intensity dependent refractive index $\mathrm{n}=\mathrm{n}_{0}+\mathrm{n}_{2} * \mathrm{I}$ :

$$
P_{\text {crit }}=\frac{3.77 \cdot \lambda^{2}}{8 \cdot \pi \cdot n_{0} \cdot n_{2}}
$$

To our knowledge for water, no data for $\mathrm{n}_{2}$ are published for ns (355 nm), ps (355 nm) and fs (343 nm) laser radiation. Wilkes et al. [37] measured $\mathrm{n}_{2}=1.9 * 10^{-16} \mathrm{~cm}^{2} / \mathrm{W}$ for $815 \mathrm{~nm}$, 90 fs pulses and $n_{2}=1.7 * 10^{-16} \mathrm{~cm}^{2} / \mathrm{W}$ for $403 \mathrm{~nm}, 250 \mathrm{fs}$ pulses. Assuming the $403 \mathrm{~nm} \mathrm{n}$ value critical powers of 0.8 MW and $0.77 \mathrm{MW}$ are estimated for $355 \mathrm{~nm}$ and $343 \mathrm{~nm}$. So, for the ns-UV laser no self-focusing is expected. For the psUV-laser weak self-focusing is possible, while the fs-UV-laser will show strong self-focusing.

For the green ps- and fs-laser wavelengths, the maximum peak powers used in this work are 2.6 MW (ps) and 253 MW (fs). Critical powers for self-focusing are estimated by $1.9 \mathrm{MW}$ at $532 \mathrm{~nm}$ and $1,7 \mathrm{MW}$ at $515 \mathrm{~nm}$, assuming $\mathrm{n}_{2}=1.7 * 10^{-16} \mathrm{~cm}^{2} / \mathrm{W}$. So, self-focusing is negligible for the green ps-laser. The green fs-laser will be affected by selffocusing [38].

For the intended applications, a long working distance between the focusing lens and the water surface and hence a low numerical aperture is required. A liquid layer thickness of a few mm above the nanofiber fleece sample is necessary. 
The liquid surface will be in the narrow section of the beam with high intensities. In case of the fs-laser the focus position and the optical breakdown position be will lifted by self-focusing to a position closely under the water surface away from the nanofiber sample. Clean cutting or structuring with the UV- and green fs laser wavelengths of immersed nanofiber flakes is therefore hard to achieve in this setup [39]. So, in this work structuring of immersed fiber samples were done only with the ps-UV- and the green ps-laser and with the ns-UV laser. The change in the focal position by the water layer of thickness $h_{0}$ above the sample was considered by lowering the sample stage by

$$
\Delta z=h_{0} \cdot\left(\frac{1}{n_{w}}-1\right)
$$

with the refractive index of water at the laser wavelength [38].
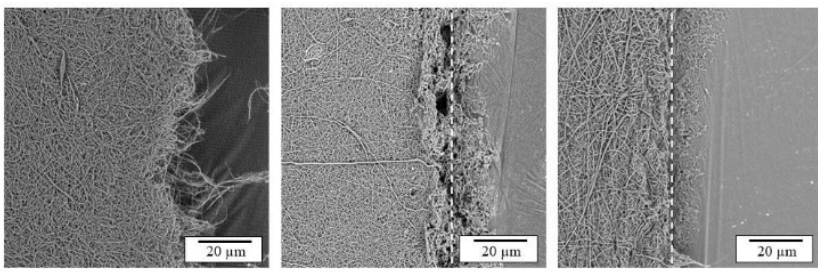

Fig. 17 Laser cuts of immersed nanofiber nonwovens. Left: ns-UV laser $F=47.3 \mathrm{~J} / \mathrm{cm}^{2}, \mathrm{f}_{\text {rep }}=200 \mathrm{~Hz}, \mathrm{~V}_{\mathrm{scan}}=0.2 \mathrm{~mm} / \mathrm{s}, \mathrm{N}_{\mathrm{E}}=5$; Center: ps-UV laser $\left(4.2 \mathrm{~J} / \mathrm{cm}^{2}, 200 \mathrm{kHz}, 10 \mathrm{~mm} / \mathrm{s}, \mathrm{NE}=40\right)$; Right: green ps-laser $\left(3.15 \mathrm{~J} / \mathrm{cm}^{2}, 200 \mathrm{kHz}, 5 \mathrm{~mm} / \mathrm{s}, \mathrm{NE}=40\right)$. The features at the right side of the dotted lines are due to SEM sample preparation. (see text)

SEM images of laser cuts under immersed conditions are shown in Fig. 17. The cutting edges obtained with the ns-UV (Fig. 17, left) show some unravelling of the fibers, but otherwise only a small laser affected zone is formed.

Cutting edges obtained with the ps-UV-laser are shown in Fig 17 (center). During drying of the sample for electron microscopy some shrinking of the nanofiber flakes on the SEM sample holders was observed, which is attributed to the SEM preparation and not to the laser process. As indicated by the dotted lines in Fig. 17, the bottom fiber layer stick on the adhesive film while the top layers contract by a few micrometres. The nonwoven shows a small laser affected zone but no unravelling of the fibers.

The cuts produced by the green ps-laser (Fig. 17, right) show the clearest edges without a significant laser affected zone.

For further improvements of cutting immersed nanofiber nonwovens laser filamentation has to be tested for the fslasers, where the power is much higher than the critical power for self-focusing [40, 41].

The 193-nm excimer laser is not well suited for nanofiber flake ablation under saline or nutrient solutions. For a $0.9 \%$ sodium chloride solution the linear absorption coefficient is $81 \mathrm{~cm}^{-1}$ corresponding to a penetration depth of $123 \mu \mathrm{m}$ [42], which is much smaller than the thickness of the liquid layer used for these applications. In pure water (absorption coefficient $0.012 \mathrm{~cm}^{-1}$, penetration depth $8.3 \mathrm{~cm}$ ), ablation is possible but easily hindered by contamination of the water through the material removal process.

\section{Conclusion}

For structuring or cutting or of small PA nanofiber flakes with dimensions smaller than a few millimeters laser affected zones must be reduced to a minimum. For dry nanofiber flakes this can be done with green ps- or fs- laser pulses. Because of the simpler technology, the ps-lasers are more economical for these purposes. For less demanding cutting processes for nanofiber nonwovens the use of UV ns-laser or green ns-lasers has to be investigated further. In terms of ablation rates and minimum structure sizes or laser affected zones, there is no advantage in using ps-UV - or fs-laser pulses.

The best processing quality of wet nanofiber samples soaked with liquids used in cell culturing is obtained with green fs-laser pulses. The ablation rates for the green fs- and the green ps-lasers are comparable. In contrast to the ablation of dry samples cutting edges on the wet samples show forming of curls of a few $\mu \mathrm{m}$ width along the edges.

Optimum processing quality for immersed PA nanofiber fleece was obtained with the green ps-laser.

Currently, cell cultivation experiments with human 3T3fibroblasts are under way to identify optimum laser generated geometric structures for efficient cell growth with respect to further development of 3D electrospun nanofiber scaffolds.

\section{Acknowledgments}

This work was carried out within the program "Forschung an Fachhochschulen - Förderlinie Ingenieurnachwuchs “, FKZ: 03FH017IA5 and funded by the Federal Ministry of Education and Research (BMBF). We are grateful to our student Denis Wiegandt for his support in several experiments and to Matthias Menzel for contributing some SEM-images. We also thank Topag Lasertechnik GmbH, Darmstadt (Germany) for supporting us with the Carbide laser system.

\section{References}

[1] P. Lesný, J. de Croos, M. Přádný, J. Vacík, J. Michálek, S. Woerly, E. Syková: Journal of Chemical Neuroanatomy, 23 (2002) 243.

[2] S. Koch, T. C. Flanagan, J. S. Sachweh, F. Tanios, H. Schnoering, T. Deichmann, V. Ella, M. Kellomaki, N. Gronloh, T. Gries, R. Tolba, T. Schmitz-Rode, S. Jockenhoevel: Biomaterials, 31 (2010) 4731.

[3] A. S. Asran, K. Razghandi, N. Aggarwal, G. H. Michler, T. Groth: Biomacromolecules, 11 (2010) 3413.

[4] S. Agarwal, J. H. Wendorff, A. Greiner: Polymer, 49 (2008) 5603.

[5] Z.-M. Huang, Y.-Z. Zhang, M. Kotaki, S. Ramakrishna: Composites Science and Technology, 63 (2003) 2223.

[6] W. Albrecht, H. Fuchs, W. Kittelmann (Eds.): "Nonwoven Fabrics" (Wiley-VCH Verlag GmbH \& Co. KGaA, Weinheim, FRG, (2002).

[7] A.K. Haghi (Ed.): "Electrospun nanofibers research: Recent developments" (Nova Science Publishers, New York, (2009).

[8] A. H. Hekmati, N. Khenoussi, H. Nouali, J. Patarin, J.-Y. Drean: Textile Research Journal, 84 (2014) 2045. 
[9] A. Greiner, J. H. Wendorff: Angew. Chem., 119 (2007) 5770.

[10] B. L.-P. Lee, H. Jeon, A. Wang, Z. Yan, J. Yu, C. Grigoropoulos, S. Li: Acta Biomater, 8 (2012) 2648.

[11] E. Adomavičiūte, T. Tamulevicius, L. Simatonis, E. Fataraitè-Urbonienè, E. Stankevicius, S. Tamulevicius: Materials Science, 21 (2015).

[12] H. W. Choi, J. K. Johnson, J. Nam, D. F. Farson, J. Lannutti: J. Laser Appl., 19 (2007) 225.

[13] M. S. Kim, J. Son, H. Lee, H. Hwang, C. H. Choi, G. Kim: Curr. Appl. Phys., 14 (2014) 1.

[14] H. Zernetsch, A. Kern, P. Jäschke, B. Glasmacher: Int J Artif Organs, 38 (2015) 607.

[15] D. Basting, G. Marowsky (Eds.): "Excimer Laser Technology" (Springer-Verlag Berlin Heidelberg, Berlin, Heidelberg, (2005).

[16] J. Ihlemann: "Laser Processing of Materials" (Springer Berlin, Heidelberg, 2010), pp. 169.

[17] K. S. Lau, P. W. Chan, K. W. Yeung, K. Chan, W. Z. Gong: J. Mat. Processing Tech., 63 (1997) 524.

[18] T. Bahners: Opt. and Quant. Elec. (1995) 1337.

[19] S. Henning, A. Heilmann, S. Schwan, A. Friedmann, H. J. Meisel, T. Ganey, C. Herbst, G. Hillrichs: Patent WO2014206755A1 (2014).

[20] J. M. Liu: Opt. Lett., 7 (1982) 196.

[21] U. Engelhardt, J. Hildenhagen, K. Dickmann: Laser Tech. J., 8 (2011) 32.

[22] M. G. Gediminas Raciukaitis: ICALEO 2005.

[23] Y. C. Lim, J. Johnson, Z. Fei, Y. Wu, D. F. Farson, J. J. Lannutti, H. W. Choi, L. J. Lee: Biotechnol. Bioeng., 108 (2011) 116.

[24] C. Park, R. Xue, J. J. Lannutti, D. F. Farson: Mater Sci Eng C Mater Biol Appl, 65 (2016) 232.

[25] M. Götze, O. Krimig, T. Kürbitz, S. Henning, A. Heilmann, G. Hillrichs: Phys. Procedia, 83 (2016) 147.

[26] S. S. Sreedhara, N. R. Tata: J. Engineered Fibers and Fabrics (2013) 132.

[27] J. Krüger, H. Niino, A. Yabe: Appl. Surf. Sci., 197-198 (2002) 800 .

[28] J. Yip, K. Chan, K. Sin, K. Lau: Mat. Research Innovations, 6 (2002) 44.

[29] J. Yip, K. Chan, K. M. Sin, K. S. Lau: Appl. Surf. Sc., 253 (2006) 2637.

[30] M. Gedvilas, G. Raiukaitis: Proc. SPIE Vol. 6157 (2006).

[31] A. Ben-Yakar, R. L. Byer: J. Appl. Phys., 96 (2004) 5316.

[32] M. Götze, O. Krimig, T. Kürbitz, S. Henning, A. Heilmann, G. Hillrichs: Proc SPIE (2017), 100940R.

[33] W. Lauterborn, A. Vogel: "Bubble Dynamics and Shock Waves" (Springer, Berlin, Heidelberg, 2013), pp. 67.

[34] P. A. Quinto-Su, M. Suzuki, C.-D. Ohl: Sci Rep, 4 (2014) 5445.

[35] A. Vogel, J. Noack, K. Nahen, D. Theisen, S. Busch, U. Parlitz, D. X. Hammer, G. D. Noojin, B. A. Rockwell, R. Birngruber: Appl. Phys. B, 68 (1999) 271.

[36] W. Liu, O. Kosareva, I. S. Golubtsov, A. Iwasaki, A. Becker, V. P. Kandidov, S. L. Chin: Appl. Phys. B, 76 (2003) 215.
[37] Z. W. Wilkes, S. Varma, Y.-H. Chen, H. M. Milchberg, T. G. Jones, A. Ting: Appl. Phys. Lett., 94 (2009) 211102.

[38] A. Menéndez-Manjón, P. Wagener, S. Barcikowski: J. Phys. Chem. C, 115 (2011) 5108.

[39] A. Vogel, J. Noack, G. Hüttman, G. Paltauf: Appl. Phys. B, 81 (2005) 1015.

[40] S. Butkus: JLMN, 9 (2014) 213.

[41] M. Malinauskas: JLMN, 10 (2015) 222.

[42] G. T. Dair, R. A. Ashman, R. H. Eikelboom, F. Reinholz, P. P. Van Saarloos: Arch. Ophthalmology, 119 (2001) 533.

(Received: June 1, 2017, Accepted: November 20, 2017) 